\title{
Eine unpublizierte Grabinschrift eines cornicen aus Ladenburg / Lopodunum
}

\author{
Francisca Feraudi-Gruénais
}

Im inschriftlichen Gesamtbefund der Provinz Germania superior war die Nennung eines cornicen auf einer Grabinschrift bislang nicht bekannt. ${ }^{1}$ Dieses Bild muß nunmehr aufgrund der Identifizierung einer bereits seit längerem bekannten, stark beschädigten Sandsteininschrift (Abb. 1) aus disparater Befundlage revidiert werden. ${ }^{2}$

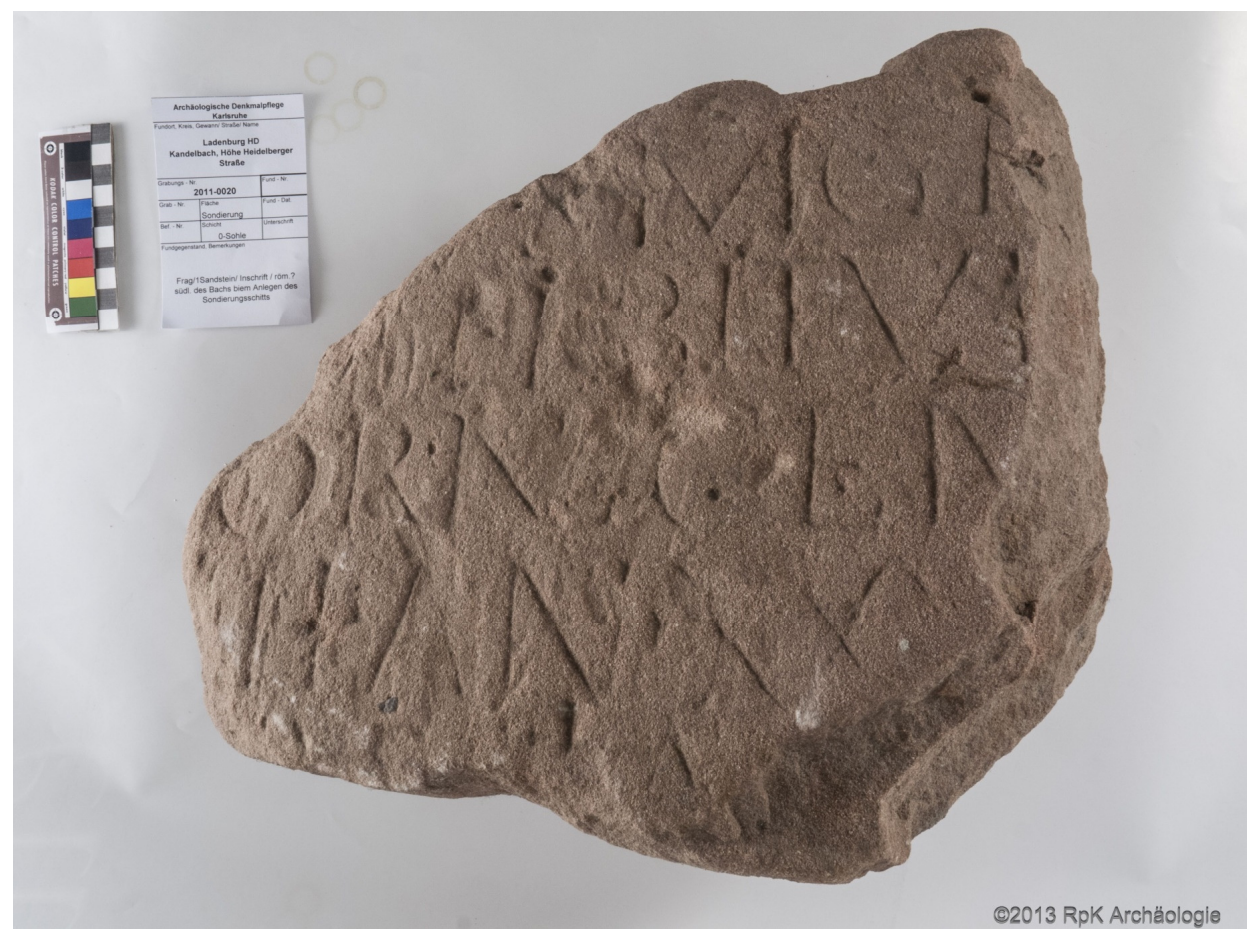

Abb. 1: Unpubliziertes Inschriftenfragment aus Ladenburg (Kandelbach)

1 Insgesamt sind cornicines inschriftlich nur rund 45 Mal bezeugt (EDCS, Suchabfrage vom 25.04.2018). Zuzüglich der Auflistungen der laterculi beläuft sich die Gesamtzahl an Erwähnungen von Soldaten dieser Funktion reichsweit auf gut 100; vgl. C.-G. Alexandrescu, Blasmusiker und Standartenträger im römischen Heer. Untersuchungen zur Benennung, Funktion und Ikonographie (Cluj-Napoca 2010) 48 Tab. 5. - Obwohl der größte Teil davon in Gestalt von Grabinschriften überliefert ist, fand sich unter diesen bislang kein einziges Beispiel aus der Provinz Germania Superior, während aus den benachbarten Provinzen Germania Inferior (CIL XIII 7779) und Belgica (P. Flotté, in: Carte archéologique de la Gaule [CAG] 57, 2, 194-195, Nr. 61) immerhin jeweils ein Beleg angeführt ist.

2 Britta Rabold, Gebietsreferentin Archäologische Denkmalpflege am Regierungspräsidium Stuttgart, Dienstsitz Karlsruhe, sei an dieser Stelle für das Überlassen dieses Steins für die wissenschaftliche Dokumentation und Auswertung gedankt. Mein Dank für logistische Hilfestellungen geht ferner an Peter Eule (Regierungspräsidium Stuttgart, Dienstsitz Karlsruhe) und Renate Ludwig (Kurpfälzisches Museum Heidelberg). Schließlich sei den Kollegen, Doktoranden und Studierenden der „Epigraphischen Werkstatt“ des Arbeitsbereichs Alte Geschichte der Universität Hamburg für die Diskussion meiner Lesung und Rekonstruktion gedankt; ebenso Krešimir Matijević (Flensburg) und Peter Probst (Hamburg) für wertvolle Anregungen und Hinweise. 
Es handelt sich um ein ringsum gebrochenes Fragment aus rotem Buntsandstein mit den Abmessungen (31) x (38) x (19) cm. Die Rückseite ist unregelmäßig bestoßen, die Vorderseite geglättet und mit einer Inschrift versehen. Die Buchstabenhöhen betragen (Z. 1) 5 / (Z. 2) 4,5 / (Z. 3) 4,5-5 / (Z. 4) 5-5,5 cm. Gefunden wurde der Stein im September 2009 in Ladenburg (Rhein-Neckar-Kreis) / Lopodunum (Germania superior) bei Baggerarbeiten am heutigen Kandelbach, in der Antike einem breiteren Neckararm, auf Höhe der Heidelberger Straße (Abb. 2). ${ }^{3}$

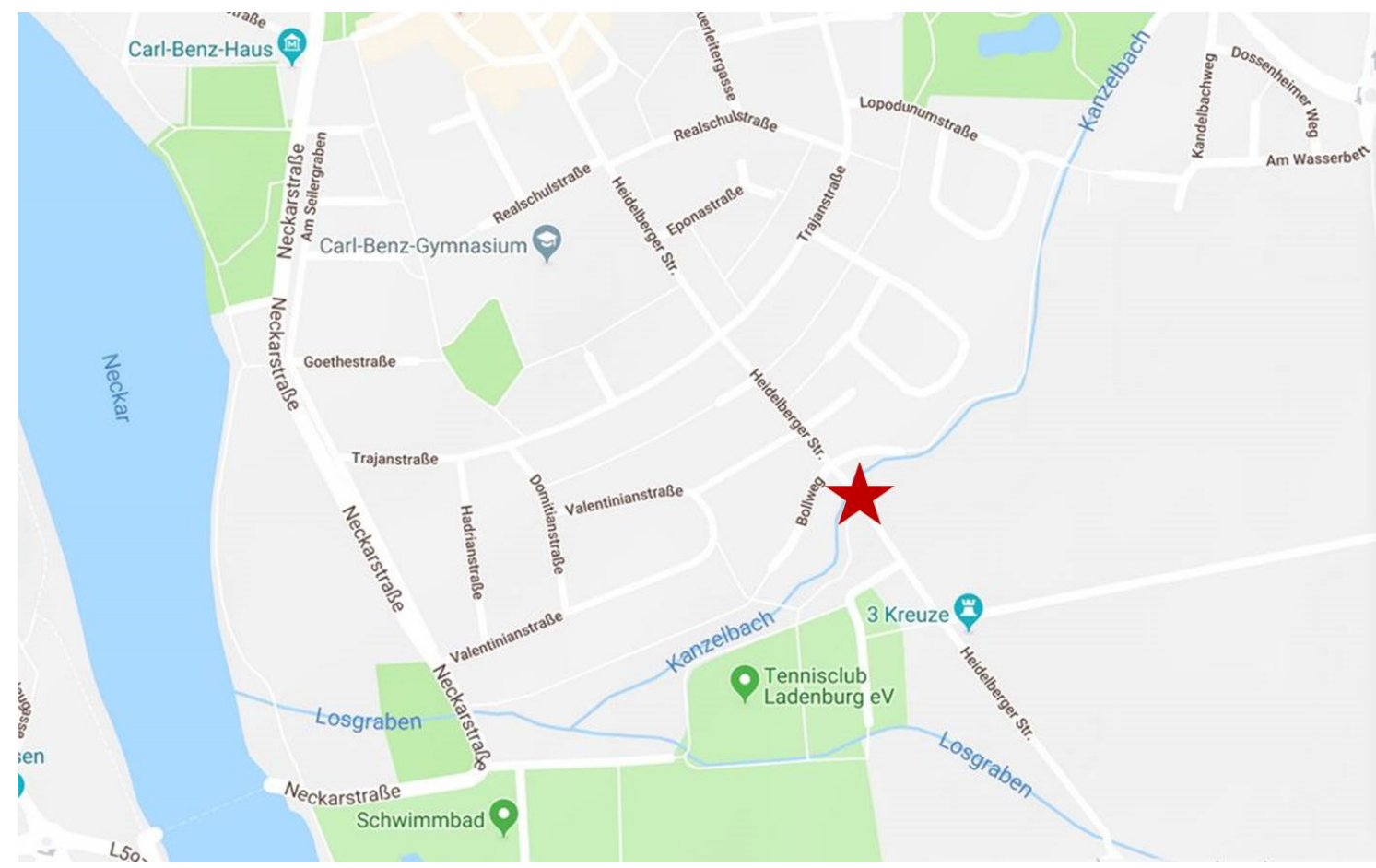

Abb. 2: Fundstelle des Ladenburger Inschriftenfragments am Kandelbach [Kanzelbach] im Bereich Heidelberger Straße

Der Erhaltungszustand der Inschrift ist von erheblichen Auswaschungen beeinträchtigt, die offenbar bedingt waren durch eine längere Lagerung des Steins im unmittelbar an der Fundstelle verlaufenden Kandelbach. ${ }^{4}$ Von der Inschrift sind fünf jeweils unvollständige Zeilen erhalten; von der letzten Zeile sind nur noch die oberen Buchstabenpartien erkennbar.

Eine eindeutige und gesicherte Lesung war aufgrund des geschilderten Zustandes der Inschrift weder auf fotografischer Basis noch per Autopsie auf Anhieb zu gewinnen und erforderte mehrfache Anläufe mit unterschiedlichen Herangehensweisen. So hat sich der Zugang über digitale Fotografien mit ihren Möglichkeiten der Vergrößerung und unterschiedlichen Kontrastierung schlecht erhaltener Partien als sehr hilfreich erwiesen; dies führte zur folgenden ersten Lesung (Abb. 3):

3 Fläche 1806/1807, Grabungs-Nr. 2009-020, Bef.-Nr. 371, Fund-Datum 09.2009, „gefunden beim Abbaggern mit großem Bagger. Daher nicht genaue Fundlage, sondern dem ca. 2-3 Meter entfernten Profil 337, Schicht 5/6 einzufügen, ..." (Notiz auf dem Fundzettel von Peter Eule, Grabungsleiter).

4 Freundlicher Hinweis von B. Rabold (Email vom 26.04.2018). 
[---] V S M A G I(?) oder P(?) oder B(?) oder N(?)

[---?]

[---] / / L(?) I B I T V I(?) [---?]

[---? c]ORNICEN [---?]

[---?] S(?)TI I(?) AN • XXX(?) oder XXV(?) [---?]

[---] A R(?) T̂ I A [---?]

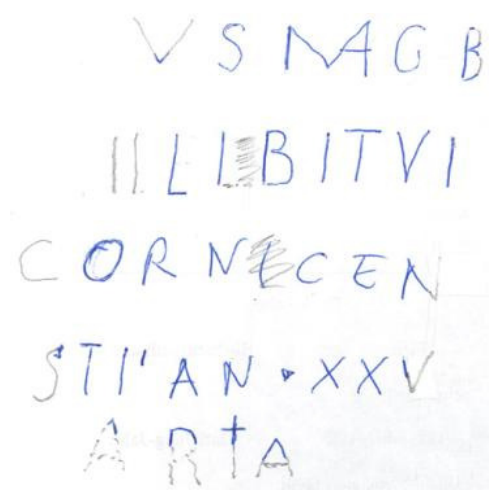

Abb. 3: Erste Textwiedergabe und Skizze vor Autopsie (auf Grundlage von Digitalfotos)

Die Lesung der rot markierten Buchstaben blieb dabei zunächst unklar und war daher in einem zweiten Schritt Gegenstand der gezielten Inaugenscheinnahme mittels Autopsie. Aus der Kombination dieser beiden Verfahren hat sich für die Zeilen 1 und 3-5 schließlich die nachfolgende zweite Lesung ergeben (Abb. 4):

[---] V S M A G N [---?]

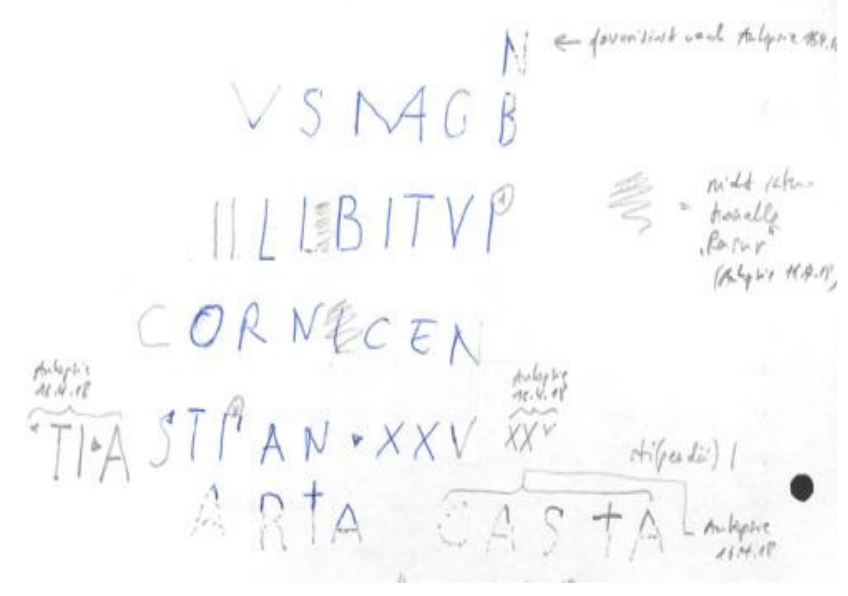

$[---?$ c]ORNICEN [---?]

$[---?] \mathbf{S T I} \bullet \mathrm{AN} \cdot \mathrm{XXV}[---?]$

[---] A S T̂ I A [---?]

Abb. 4: Optimierte Textwiedergabe nach Kombination mit Autopsie

Somit konnten in Z. 1 die allein schon aussprachetechnisch unwahrscheinlichen Optionen P und B definitiv ausgeschlossen und die Senkrechthaste am rechten Rand eindeutig als Teil des Buchstabens $\mathbf{N}$ identifiziert werden.

Des Weiteren ist die Kerbe zu Beginn von Z. 4 sicher als die obere Serife des Buchstabens S auszumachen, während die Meißelspur nach TI sicher einem Interpunktionszeichen $(\bullet)$ zuzuordnen ist und nicht einem kleiner geschriebenen I bzw. Zeichen für die Zahl eins, wie von mir anfänglich auf Grundlage des Fotos vermutet. Auszuschließen ist am Ende der gleichen Zeile die zunächst in Erwägung gezogene Option X, für die im linken unteren Teil jegliche Evidenz in Gestalt einer entsprechenden Diagonalhaste fehlt; anzunehmen ist hier daher der Buchstabe bzw. das Zahlzeichen $\mathbf{V}$, wobei unklar bleibt, ob seine untere Spitze bis auf die imaginierte Zeilenlinie der übrigen Buchstaben hinuntergereicht oder auf halber Höhe geendet hat. So jedenfalls stellt sich der Befund dar, der sich anhand der reinen Meißelspuren ergibt, und der freilich selbst bei deren nicht zerstörungsbedingtem Ausbleiben insofern immer nur begrenzt etwas über die tatsächlich gemeinte Inschrift auszusagen 
vermag, als letztlich ausschlaggebend erst die - möglicherweise auch abweichende farbige Ausmalung der Buchstaben gewesen ist.

Bleibt noch die letzte Zeile, deren Buchstaben nur im oberen Bereich erhalten sind: Der zunächst als R gelesene zweite Buchstabe (s. o. Abb. 3, erste Lesung) muss nach Autopsie und nochmaligem Abgleich mit unterschiedlich ausgeleuchteten Fotos nunmehr als $\mathbf{S}$ gelesen werden.

Auf dieser Grundlage ergibt sich für die genannten Zeilen eine weitgehend unproblematische Transkription wie folgt:

------ / [---] us M̂agn[---?] / [---] / / L(?) I B I T V I(?) [---?] / [---? c] ornicen [---?] / [---?] sti(pendi---?) an(norum) XXV [---?] / [---] A S T I A [---?] / [------?

Stolperstellen verbleiben nunmehr noch in der zweiten und fünften Zeile. Für deren sinnvolle Auflösung beziehungsweise Ergänzung reichen die bis hierhin durchgespielten unterschiedlichen Methoden einer möglichst präzisen Lesung der Buchstabenspuren allein nicht mehr aus. In den engeren Fokus müssen nunmehr auch denkmaltypologische und gattungsspezifische Aspekte einbezogen werden, sowie weitere Fragen, die sich hieraus ergeben mögen:

Hinsichtlich der Inschriftengattung ist dabei unstrittig, dass mit dem Ladenburger Fragment eine römische Grabinschrift bezeugt ist - übrigens eine von, nunmehr, insgesamt nur drei bekannten ${ }^{5}$ Inschriften des Sepulkralbereichs aus dem Territorium von Lopodunum und insofern von umso größerem Interesse. Der Ausdruck an(nnorum) $X X V$ [---?] ist hierfür ein entscheidendes Indiz. Auch die unmittelbar vorangestellte, mutmaßliche, Stipendienangabe des Verstorbenen, der innerhalb der Truppe die Funktion eines cornicen ausübte, ist ein wichtiger Hinweis. ${ }^{6}$ Dieses Faktum stellt, wie schon gesagt, insofern eine Besonderheit dar, als im epigraphischen Befund des ehemaligen Militärstandorts Ladenburg ${ }^{7}$ mit Ausnahme einer - mittlerweile verschollenen - Weihung eines decurio alae I Cannanefatium ${ }^{8}$ ansonsten kein weiteres Militärpersonal explizit bezeugt ist.

Als Denkmaltyp kann aufgrund der Abmessungen eine Grabstele angenommen werden, die im Bereich des Unteren Neckars verbreitetste Form sepulkraler Inschriftenmonumente, die auch entlang der Gräberstraße zwischen Heidelberg und Ladenburg, einem der größten Gräberfelder im römischen Deutschland mit einer gut 100 Jahre währenden Nutzungsdauer zwischen 80-190 n. Chr., mehrfach belegt sind. Oberhalb des Inschriftfeldes hätte sich in diesem Fall noch ein Relieffeld, möglicherweise mit einer Soldatendarstellung, befunden, während die Stele nach

5 Bislang bekannt: CIL XIII 11741 (R. Wiegels, Lopodunum II. Inschriften und Kulturdenkmäler aus dem römischen Ladenburg am Neckar [Stuttgart 2000] 59-61, Nr. 16; Abb. 18; EDH036402) und CIL XIII 6423 (Wiegels, ebenda 61-63, Nr. 17; Abb. 19; EDH036403).

$6 \mathrm{Zu}$ Vorkommen und Funktion der in den Legionen und unberittenen Hilfstruppen eingesetzen cornicines s. v. a. P. Cauer, EphEp 4, 1881, 376-377, Nr. 26-54; A. von Domaszewski, Die Rangordnung des römischen Heeres (Köln ${ }^{2}$ 1967) 58-59; G. Wille, Musica Romana. Die Bedeutung der Musik im Leben der Römer (Amsterdam 1967) 93-96; M. Junkelmann, Die Legionen des Augustus (München ${ }^{15}$ 2015) 154-155; Alexandrescu (w. o. Anm. 1) bes. 45-52. 257262.

7 Der Bestand an Inschriften aus Ladenburg umfasst rund 60 Belege (EDH: 85 [davon 62 ohne Schreibtechnik ,gestempelt"]; EDCS: 94 [davon 62 ohne Inschriftengattung ,sigilla impressa“"]; Suchabfrage vom 04.05.2018).

8 CIL XIII 11740; Wiegels (wie Anm. 5) 55-57, Nr. 13; Abb. 15; R. Wiegels, in: W. Spickermann (Hrsg.), Religion in den germanischen Provinzen Roms (Tübingen 2001) 199-200; EDH036400. 
unten hin durch einen grob behauenen Verankerungszapfen abgeschlossen war. Stelen dieser Art kamen auf Gesamthöhen zwischen 180 und $230 \mathrm{~cm} .^{9}$

Damit kommen wir zurück zu den noch unklaren Lesungen der Zeilen 2 und 5: Dem üblichen Aufbau von Grabinschriften folgend, ist gegen Ende der Inschrift, d. h. nach Nennung des Verstorbenen, seiner militärischen Position und seiner Lebensjahre, der Name des Dedikanten zu erwarten. In diesem Fall war dies offenbar eine Frau; ob die Mutter, Schwester oder Ehefrau des Verstorbenen bleibt offen. Der Name in Z. 5 endet auf [---]astia und kann auf Grundlage einer systematischen Durchsicht des in der Provinz Germania superior epigraphisch bezeugten Repertoriums weiblicher Namen dieser Endung in der vollständigen Form nur Castia - [C] A S T̂T I A [---?] gelautet haben. ${ }^{10}$

Weniger eindeutig stellt sich demgegenüber die Situation in Z. 2 dar. Am ehesten sind an dieser Stelle der Grabinschrift entweder weitere Namen beziehungsweise Namensbestanteile des Verstorbenen [---]us Magn[---] ${ }^{11}$ zu erwarten oder aber auf den cornicen Bezug nehmende Truppenbezeichungen.

Zur Option eines Namens bzw. Namensbestandteils lässt sich festhalten, dass der disparate Zustand der erhaltenen Buchstaben das Herauslesen von Namen nicht einfacher macht. Auch ist in dieser Region der obergermanischen Provinz stets mit lokalen, d. h. keltischen oder germanischen Namensformen zu rechnen. Immerhin kommt diesen Unwägbarkeiten die Beobachtung entgegen, dass im ersten Drittel der erhaltenen Zeile, das heißt vor „IBITVI(?)“, mit ziemlicher Sicherheit ein Interpunktionszeichen $(\bullet)$ ausgemacht werden kann. Dank dieser Zäsur, die hier methodisch als nahezu sichere Prämisse vorausgesetzt wird, darf sich die Suche nach Buchstabenfolgen, die ein (oder mehrere?) Worte ergeben müssen, auf die eindeutig lesbare Buchstabenkette „•IBITV“ konzentrieren. Das Ergebnis dieser Suche ist mit

9 Vgl. u. a. F. Feraudi-Gruénais / R. Ludwig, Die Heidelberger Römersteine. Bildwerke, Architekturteile und Inschriften im Kurpfälzischen Museum Heidelberg (Heidelberg 2017) 78-85. 87-89, Nr. 44-47. 49-50. - S. auch unten Abb. 6 (rechts).

10 Die einzige Parallele für einen auf ,--astia“ endenden Namen findet sich als Castia auf einer Inschrift aus Castrum Rauracense (Kaiseraugst, Germania superior): CIL XIII 5284; W. Faust, Die Grabstelen des 2. und 3. Jahrhunderts im Rheingebiet (Köln 1998) 115, Nr. 72; EDH076066. Ein einziger weiterer Beleg für den um eine Silbe längeren Namen „Castia-na“ aus Novae (Svištov, Moesia inferior) ist im vorliegenden Kontext nicht repräsentativ. - Zur Absicherung der Lesung der stark beschädigten Buchstaben in Z. 5 vor der gesicherten TI-Ligatur wurden sämtliche auf ,,tia" endenden Namen, die auf Inschriften der Germania superior bezeugt sind, durchgesehen. Im Einzelnen sind dies: Ioincatia, Adnamatia, Manatia, Gratia, Ratia, Speratia, Honoratia, Spectatia, Aetia, Mogetia, Avitia, Carantia, Innocentia, Prudentia, Polentia, Florentia, Reverentia, Aventia, Sequentia, Quintia, Arruntia, Aceptia, Neptia, Martia, CaStia, ReStia, Gattia. Von allen der TILigatur unmittelbar vorangehenden Buchstaben (A, E, I N, P, R, S, T) passt einzig der Buchstabe S zur erhaltenen Meißelspur, somit die Namen REstia und CAstia. Da der dem S vorangehende Buchstabe über eine nach rechts unten verlaufende Diagonalhaste verfügt, scheidet Restia aus, so dass angesichts des einzig verbleibenden Namens Castia der kaum noch lesbare Buchstabe A vor S als gesichert gelten darf.

11 Der fragmentierte Namensbestandteil Magn[---] ist nach Ausweis der in Obergermanien bezeugten mit „Mag-“ beginnenden Namen am wahrscheinlichsten auf den Namen Magnus zu ergänzen (,Mag-“ sonst durchgehend mit anschließendem I oder V kombiniert: Magianus [Gottheit], Magiatus, Magiorix, Magissa, Magius, Magurio). - Die daran anschließende Frage, ob , Magnus“ als Eigenname des Dedikanten ([--- i] us Magn[us]) oder als Patronymikon [---] us Magn[i f.]) zu verstehen ist, kann nicht mit Sicherheit beantwortet werden; aufgrund der erhaltenen Abmessungen und Proportionen des Layouts der Inschrift erscheint das Vorhandensein einer Filiationsangabe allerdings als eher unwahrscheinlich (s. auch unten S. 9).

http://www.fera-journal.eu 
rund zehn Belegen ${ }^{12}$ überschaubar, und die einzigen passenden Belege von Personennamen sind tatsächlich ausschließlich in den gallisch-germanischen Provinzen bezeugt: Relibitus (Germania inferior), Daribitus (Aquitania, Belgica, Germania inferior, Germania superior). Auch wenn das kreative Auge des Epigraphikers keine Schwierigkeiten damit hätte, in der Lücke vor „IBITV“ treffsicher die Buchstaben „DAR_“ oder noch passender „REL-““ zu sehen, bremst das Flexionsproblem am Ende der ausgemachten Namen ein solches Ansinnen aus, das mit der o-Deklination nicht vereinbar ist. Diese Option ist somit hinfällig. An dieser Stelle vermag die Funktion der online-Suche im sogenannten „Majuskeltext“ der $E D H$ als Inspirationsquelle zu dienen, da sie nämlich Abfragen nach Buchstabenketten auch über Wort- und Zeilengrenzen und editorische Eingriffe wie Auflösungen und Ergänzungen hinweg ermöglicht und gerade in diffizilen Fällen wie dem vorliegenden bislang nicht berücksichtigte Lösungsansätze in das Blickfeld rücken kann. In der Tat führt die Majuskelsuche nach „IBITV“ im epigraphischen Befund zu den zusätzlichen Namen Bituna, ${ }^{13}$ Bitua $^{14}$ und Bitus. ${ }^{15}$ Der Name Bituna könnte aufgrund der Senkrechthaste im an das $\mathrm{V}$ anschließenden möglichen Buchstaben $\mathrm{N}$ in Betracht kommen, doch lässt dieses singuläre Beispiel, zumal aus einer vergleichsweise entfernt liegenden Provinz, den Alpes Cottiae, diese Option noch nicht als erste Wahl erscheinen.

Doch nicht nur mit „BITV“ beginnende Personennamen sind Bestandteile der Namengebung, sondern auch Origonamen, die sich in der Tat mehrfach und unter anderem auch auf Inschriften Obergermaniens finden lassen, nämlich in der Form von civitas Biturigum ${ }^{16}$ und (domo / natione) Biturix ${ }^{17}$. Zudem kommt der an das V anschließende Buchstabe R mit anfänglicher Senkrechthaste dem Textbefund auf der Ladenburger Inschrift entgegen, so dass ein auf „Biturigum “ / „Biturix“ bezugnehmendes Wort nunmehr endlich als die erste ernsthaft in Erwägung zu ziehende Option erscheint.

Zur Option einer Truppenbezeichnung ist anzumerken, dass die bis hierhin aus dem Spiel mit den erhaltenen Buchstaben gewonnenen Erkenntnisse natürlich auch für die Suche nach möglichen Truppenbezeichnungen ihre Bedeutung haben. So stehen nämlich die gleichen Bituriges, ein gallischer Stamm aus der römischen Provinz Aquitania im heutigen Zentralfrankreich Pate für den Namen der in augusteischer Zeit dort ausgehobenen cohors I (Aquitanorum) Biturigum. ${ }^{18}$ Diese Kohorte ist epigraphisch ausschließlich auf Militärdiplomen und im instrumentum

12 Ein nahezu einhelliges Ergebnis bringen die Suchabfragen nach ,ibitu“ in EDCS und EDH (08.05.2018). Das Suchergebnis in $E D H$ führt noch den weiteren Namen Ibituathif (IRT 886e; HD020947) auf; die nordafrikanische Provenienz und die mit dem Ladenburger Befund nicht übereinstimmenden Buchstabenfolge „VA“ sind vorliegend jedoch eindeutige Ausschlusskriterien.

13 ...patri Bitunae ... (Grabinschrift, Alpes Cottiae; CIL XII 89; EDH065263).

14 ... [s] ervi Bitua ... (Grabinschrift, Pannonia superior; CIL III 14359, 21; EDH028219).

15 ... Septi(mius) Bitus ... (Grabinschrift, Dacia; CIL III 12598; EDH046397); ...He(r)culi / Bitus Bit(i) ... (Weihinschrift, Moesia superior; CIL III 8095; EDH032307).

16 ... ex civitate Biturigum ... (Grabinschrift, Germania superior; CIL XIII 5276; EDH076021).

$17 \ldots$ B/i]tur $<i>x \ldots$ (Weihinschrift, Germania superior; CIL XIII 6434; EDH042502); ... natione Biturix ... (Grabinschrift, Raetia; CIL III 5831; EDH058603); ... domo Biturix ... (Grabinschrift, Dalmatia; CIL III 2065; EDH063264).

18 E. Stein - E. Ritterling, Die kaiserlichen Beamten und Truppenkörper im römischen Deutschland unter dem Prinzipat (Wien 1932) 171; J. Spaul, COHORS ${ }^{2}$. The evidence for and a short history of the auxiliary infantry units of the Imperial Roman Army (Oxford 2000) 145; D. B. Cuff. The auxilia in Roman Britain and the Two Germanies from Augustus to Caracalla: Family, Religion and 'Romanization' (Diss. Toronto 2010) 250 https://tspace.library.utoronto.ca/bitstream/1807/24732/1/Cuff David B 201006 PhD thesis.pdf

http://www.fera-journal.eu 
domesticum beziehungsweise militare der Germania superior bezeugt; ihr Standort lag im heutigen Ort Langenhain (Hofheim/Taunus) am westlichen Wetteraulimes (Abb. 8). Sollte also in der zweiten Zeile des Ladenburger Fragments die militärische Einheit, in der der Verstorbene zu Lebzeiten gedient hatte, vermerkt gewesen sein, so spricht sehr viel für die genannte Kohorte. Trifft dies zu, so fände auch das dem B vorangehende, bislang als Buchstabe gelesene I seine ganz natürliche Erklärung als Nummerierung der Kohorte. Es ist nicht ausgeschlossen, dass der diesem voranstehende, von mir behelfsmäßig als $L($ ?) gelesene Buchstabe nunmehr als $H$ mit diesem wiederum voranstehendem $O \mathrm{zu}$ interpretieren ist: [miles? c] $\boldsymbol{O ( ? ) H ( ? )} \bullet I$ BITVR [---?], auch wenn dies weder per Autopsie noch mit den gewöhnlichen digitalfotografischen Methoden mit Sicherheit verifiziert werden kann.

Sämtliche soeben dargelegten Argumente zusammengenommen, wonach für Zeile 2 die Möglichkeit eines zu ergänzenden Personennamens ausgeschlossen werden musste, während für eine origo- oder eine Truppenbezeichnung deutlich konkretere Anhaltspunkte gefunden werden konnten, vermag die Option Origoname, gerade auch angesichts des nahezu sicher eingemeißelten Interpunktionszeichens vor I, letztlich weniger zu überzeugen. Somit gewinnt eine Textrekonstruktion im Sinne von cohors I Biturigum einiges an Wahrscheinlichkeit, die hier im Sinne einer ,lectio difficilior', und damit einer gewiss nicht ganz unproblematischen Version, etwas weiter ausgeführt sei.

Die Lesung des Ladenburger Inschriftenfragments präsentiert sich demnach wie folgt (Abb. 5):

$$
\text { [---]VS MAGN[---?] }
$$

[---? c]O(?)H(?) • I BITVR[igum ---?]

$[---?$ c]ORNICEN [---?]

$[---?] \mathrm{STI} \bullet \mathrm{AN} \cdot \mathrm{XXV}[---?]$

[---? C]AST̂IIA [---?]

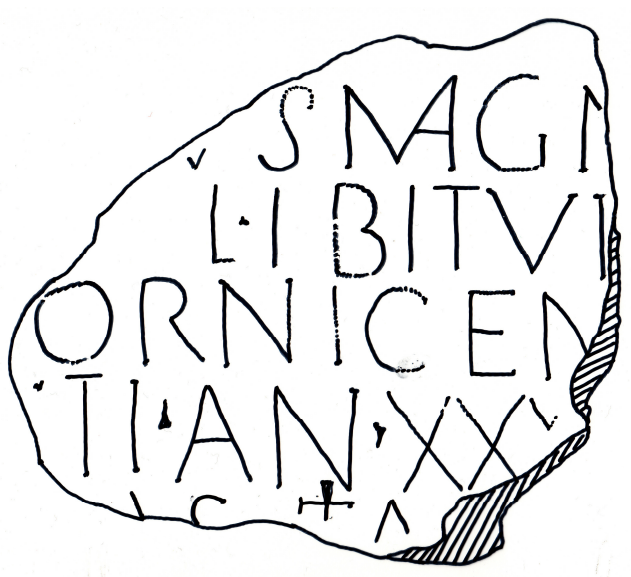

$-----\cdot$

------ / [---] us M̂agn[---?] / [---? c]o(?)h(?)(ortis) I Bitur[igum ---?] / [---? c]ornicen [---?] / [---?] sti(pendii) an(norum) XXV [---?] / [--- C] astia [---?] / [-----?

Abb. 5: Lesung / Rekonstruktion und Umzeichnung des Ladenburger Inschriftenfragments vom Kandelbach

In dieser Wiederherstellung weist das Fragment alle wesentlichen Elemente einer Grabinschrift auf. Der am rechten Rand durch eckige Klammern vorsorglich einkalkulierte Platz erscheint nunmehr als verzichtbar, der Name der Kohorte war möglicherweise einfach abgekürzt eingemeißelt. Auch ist in Zeile 4 nach dem Zahlzeichen V der Lebensjahrangabe in Anbetracht von erst einem Dienstjahr kaum 
ein weiteres Zahlzeichen anzunehmen. ${ }^{19}$ Die Stipendienangabe ohne explizite Angabe der Zahl, die in Analogie zu (nicht explizit genannten) einjährigen Lebensalterdaten dann wohl als $I$ zu verstehen wäre, ${ }^{20}$ bleibt mit der Ladenburger cornicen-Inschrift vor dem Hintergrund des insgesamt sehr überschaubaren Bestandes an einjährigen Dienstangaben bislang ohne Vergleich. ${ }^{21}$ Oberhalb des Namens des verstorbenen, ursprünglich möglicherweise als miles bezeichneten Soldaten darf wohl noch eine Zeile mit der Weiheformel $D M$ erwartet werden, im Anschluss an die letzte Zeile eventuell eine Widmungsformel wie coniux $F C$, so dass sich die rekonstruierte Inschrift abschließend folgendermaßen darstellt:

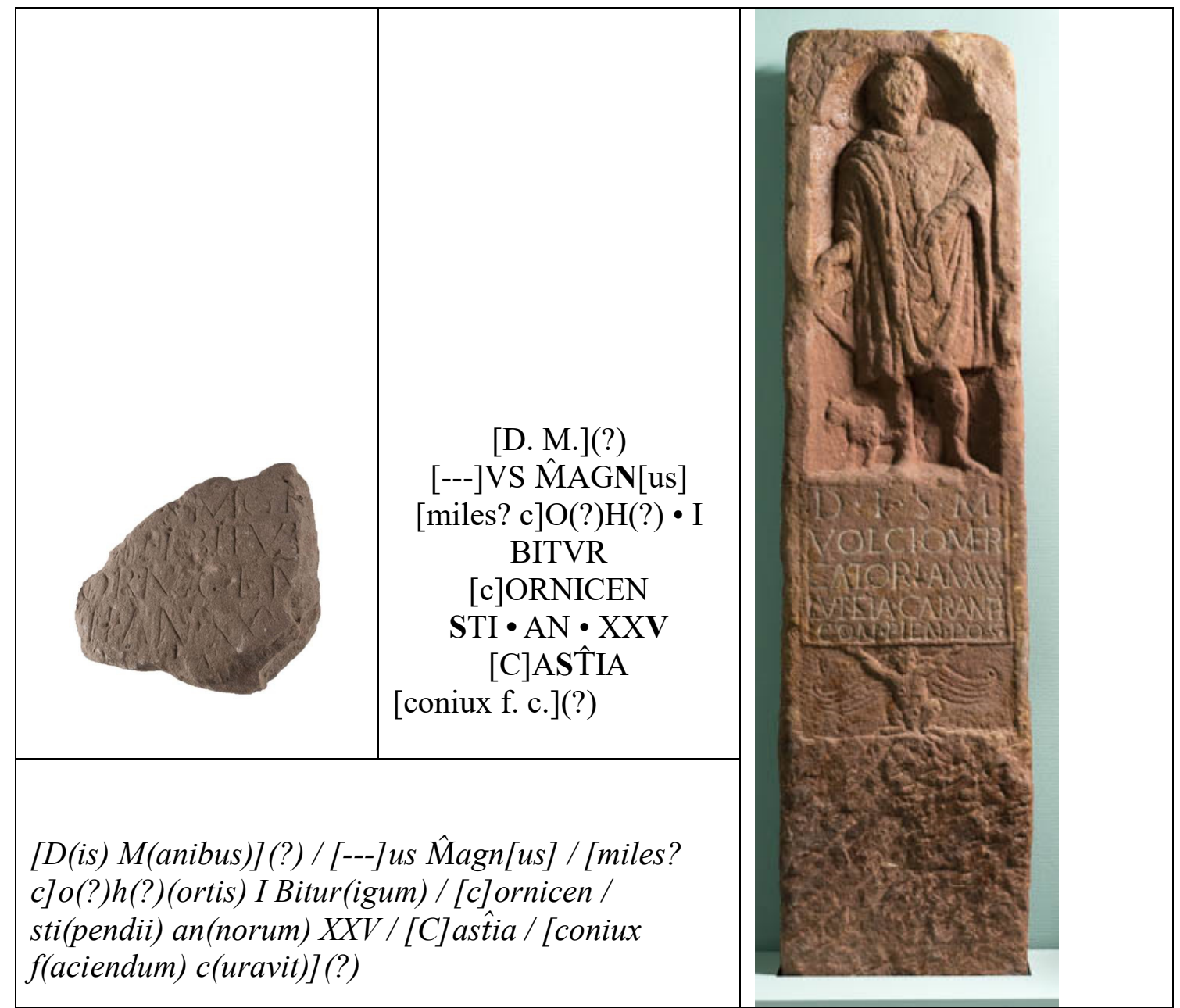

Abb. 6: Fragment der Ladenburger cornicen-Inschrift mit Textrekonstruktion (links) und die Grabstele des Volcius Mercator aus Heidelberg (s. Anm. 22) als typologisches und ähnlich dimensioniertes Vergleichsstück (rechts)

19 Die Angabe von nur einem Stipendium ist im über EDCS und EDH (Suchabfrage vom 04.05.2018) greifbaren epigraphischen Befund bislang nur sechs Mal bezeugt, dabei kombiniert mit Lebensalterangaben zwischen 18 bis maximal 25 Jahren und bezogen auf Legionssoldaten. Bemerkenswerterweise stammen gleich fünf davon - dort allerdings, anders als beim Ladenburger Stück, mit der Abkürzung stip. I - aus der Provinzhauptstadt Moguntiacum (Mainz, Germania superior; CIL XIII 6893. 11853-11856). Das sechste, ähnlich wie in Ladenburg mit sti. + I abgekürzte, Beispiel ist aus Burnum (Dalmatia; CIL III 15004) belegt.

20 anno oder annum ohne den Zusatz I bzw. uno oder unum ist in den lateinischen Inschriften der beiden Germanien, Italiens, Nordafrikas, der Donauprovinzen und des östlichen Mittelmeerraumes sehr geläufig.

21 Vgl. o. Anm. 19. 
Für den Inschriftträger ergeben sich aus der oben dargelegten Rekonstruktion des Textes Parameter, die für die ursprünglichen Abmessungen eine eineinhalbfache rekonstruierte Breite postulieren - im konkreten Fall (31) $\mathrm{cm}+$ ca. $15 \mathrm{~cm}-$, was an eine der eher kleiner dimensionierten Grabstelen denken lässt, wie sie mehrfach aus den benachbarten Heidelberger Grabarealen bekannt sind. Unmittelbar vergleichbar ist etwa die Grabstele des Volcius Mercator aus Heidelberg-Bergheim aus dem ersten Drittel des 2. Jh. n. Chr. mit einer Höhe von 185, einer Breite von 46, einer Tiefe von $20 \mathrm{~cm}$ und sehr ähnlichen Buchstabenhöhen zwischen 5,5 und 2,5 cm (Abb. 6). ${ }^{22}$

Somit bleibt die Frage der Datierung: Der einzig verwertbare Anhaltspunkt ist hierfür die wiedergewonnene Lesung des Kohortennamens. Militärdiplome belegen die Aktivität der cohors I Biturigum in Obergermanien zwischen 74 und 134 n. Chr.; ${ }^{23}$ tesserae aus dem Kohortenstandort Kastell Langenhain bezeugen ihre Existenz noch in den Jahren 180/192 n. Chr. ${ }^{24}$ und wohl auch darüber hinaus bis ins mittlere 3. Jh. n. Chr. Hieraus ergibt sich eine grobe zeitliche Einordnung des Ladenburger Inschriftenfragments vom ausgehenden 1 . bis mindestens in das ausgehende 2 . Jh. n. $\mathrm{Chr}^{25}$

Zuletzt gilt es nach der Bedeutung des Fundes dieser Inschrift in Ladenburg zu fragen: Dass ein zeitweiliger Garnisonsort wie Lopodunum, der im letzten Drittel des 1. Jhs. n. Chr. nacheinander über zwei Kastelle verfügte, ${ }^{26}$ als einzig gesicherte militärische Einheit die ala I Cannanefatium beheimatete und um 100 n. Chr. nach Auflassung des Lagers zum Zentralort der civitas Sueoborum Nicrensium mit dem Beinamen Ulpia erhoben worden ist, auch letzte Ruhestätte dort stationierter oder beheimateter Soldaten war, ist an sich nichts Besonderes. Durchaus bemerkenswert ist jedoch, dass vom Territorium Ladenburgs bisher nur zwei Grabinschriften aus dem 2./3. Jh. n. Chr. bekannt sind; beide Verstorbene, ein libertus und ein dispensator, waren Zivilisten. Das neue Inschriftenfragment belegt somit erstmals im epigraphischen Repertorium Ladenburgs überhaupt die Bestattung eines Militärangehörigen. Zudem ist ein solcher, hier ein immunis in der spezifischen Funktion eines cornicen, wie bereits angesprochen, im sepulkralinschriftlichen Befund der gesamten Provinz Germania superior bislang nicht bezeugt. ${ }^{27}$ Angesichts all dieser an Einzigartigkeiten zwar reichen, sich in ihrem geo- bzw. topographischen Kontext jedoch allzu disparat darstellenden Gegebenheiten erscheint eine entsprechend $\mathrm{zu}$ stützende genauere historische Interpretation des neu identifizierten Ladenburger cornicen kaum möglich. Die Fundstelle der Grabinschrift unmittelbar

22 CIL XIII 6410; EDH036468; Feraudi-Gruénais / Ludwig (w. o. Anm. 9) 78-79, Nr. 44.

23 CIL XVI 20 (74 n. Chr.); CIL XVI 36 (90 n. Chr.); RMD 90 (129/30 n. Chr.); CIL XVI 80 (134 n. Chr.); s. auch Stein - Ritterling und Spaul (w. o. Anm. 18); J. Scheuerbrandt, Exercitus: Aufgaben, Organisation und Befehlsstruktur römischer Armeen während der Kaiserzeit (Diss. Freiburg 2004) Tab. 3 <URN: urn:nbn:de:bsz:25-opus-71774>.

24 AE 1992, 1291 und 1293-1295; EDH013918, EDH038761, EDH038765, EDH038768.

25 Merkmale im Formular wie die mutmaßliche Weiheformel an die Totengötter, die Nennung des Verstorbenen im Nominativ oder die Stipendienangabe bieten angesichts ihrer langen Verwendung in der epigraphischen Praxis der Germania superior keine hinreichenden Indizien für eine engere Datierung. - Für eine möglicherweise inhaltlich zu begründende zeitliche Eingrenzung s. u. S. 12.

26 Um 73 n. Chr. Rasen-Soden-Kastell am Neckarufer („Kastell II“), ab 74 n. Chr. Holz-Erde-Kastell etwas südlich gesichert („Kastell I“); nach seiner Zerstörung wohl in Zusammenhang mit dem Saturninus-Aufstand 89 n. Chr. als Steinkastell neu errichtet. Als (zeitweiliger) Standort der ala I Cannanefatium belegt durch eine Weihinschrift des Dekurionen L. Gallionius Ianuarius (s. o. Anm. 8).

27 S. dazu o. Anm. 1. 
außerhalb der Stadtmauer an der Ausfallstraße nach Heidelberg (s. auch o. Abb. 2) liefert ferner außer dem sich hieraus ergebenden Hinweis, dass die Grabstele eben von dieser Gräberstraße stammen wird und es sich somit um keine Verschleppung handeln dürfte, keinerlei weiterführende Anhaltspunkte (Abb. 7). ${ }^{28}$ Möglicherweise war der Grabstein in das Bett des seinerzeit hier noch vorbeifließenden und später verlandeten Neckararms gestürzt.

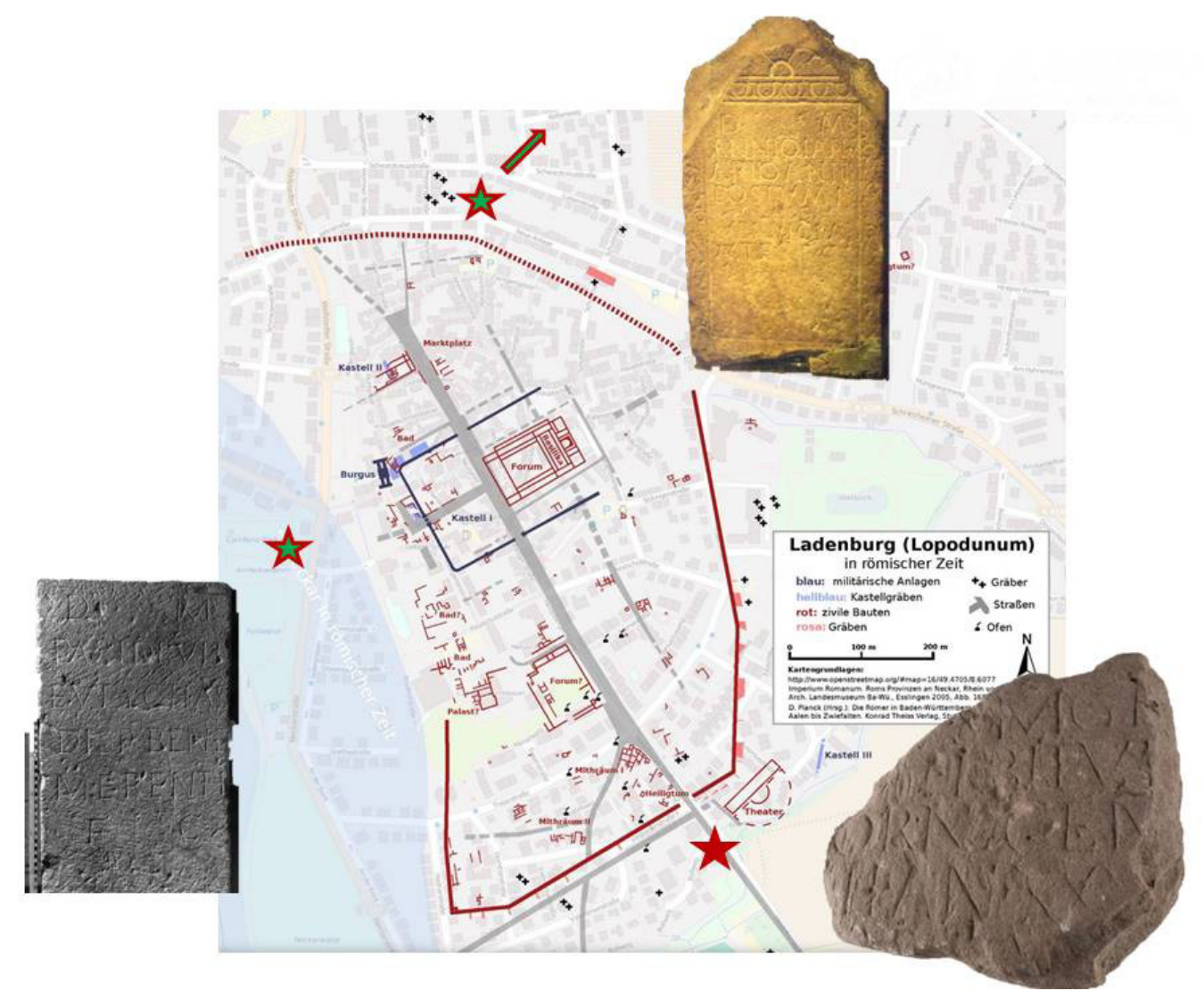

Abb. 7: Fundstelle der cornicen-Inschrift im Plan des antiken Lopdunum (unten) sowie der beiden einzigen weiteren bisher von dort bekannt gewordenen Grabinschriften (s. o. Anm. 5).

Auch wenn man sich nun damit abfinden muss, dass aus der mangelnden Kontextualisierung der Stele derzeit keine weiterführenden Erkenntnisse zu gewinnen sind, lässt eine Frage doch keine Ruhe: Welchen Bezug hatte dieser cornicen zu Ladenburg? An dieser Stelle kann nur spekuliert werden; doch scheint mir die Dislokationsgeschichte des am westlichen Wetteraulimes stationierten Truppenverbandes, dem die in Langenhain stationierte cohors I Biturigum angehörte, einen Anhaltspunkt zu geben, der zumindest für eine Hypothese taugen mag.

28 „Leider erlaubt auch der Fundkontext keine weiterführenden Aussagen.“ (B. Rabold, Email vom 26.4.2018). 


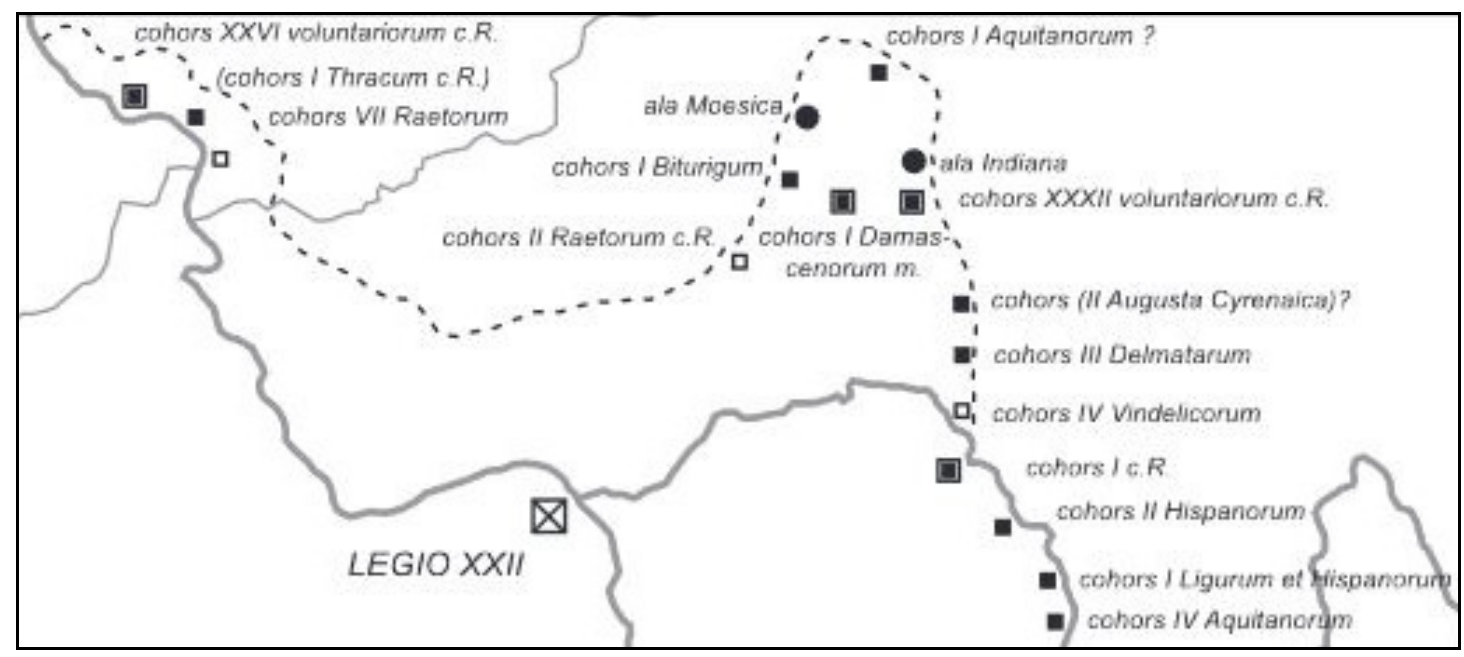

Abb. 8: Dislokation der Truppenverbände am Wetteraulimes

$\mathrm{Zu}$ diesem Verband zählte nämlich $\mathrm{u}$. a. auch die in Butzbach stationierte cohors II Raetorum. (Abb. 8) - jedenfalls bis 135 n. Chr., als sie auf die Saalburg versetzt wurde und an ihrer Stelle die cohors II Augusta Cyrenaica nach Butzbach kam, wo sie bis $150 \mathrm{n}$. Chr. verblieb. ${ }^{29}$ Diese kyrenäische Kohorte war zuvor im Westkastell von Heidelberg-Neuenheim stationiert, das noch militärisch genutzt wurde, als Ladenburg spätestens seit dem frühen 2. Jh. n. Chr. bereits zu einem reinen Zivilort geworden war. Es kann daher nicht ausgeschlossen werden, dass der Ladenburger cornicen zunächst als Soldat der cohors II Augusta Cyrenaica mit dieser von Heidelberg in die Wetterau gekommen war und, innerhalb des dortigen Truppenverbandes als cornicen Eingang in die cohors I Biturigum fand. Nach seinem recht bald erfolgten Tod, wäre er in seiner rund $120 \mathrm{~km}$ entfernten Heimatstadt (Abb. 9), eben mutmaßlich Ladenburg vor den Toren Heidelbergs, das über die Römerstraße gerade einmal 4 leugae $=8,8 \mathrm{~km}$ entfernt ist, bestattet worden. ${ }^{30}$ Trifft diese Rekonstruktion zu, ließe sich die Datierung des Grabsteins gut auf die Jahre 135/136 n. Chr. eingrenzen.

29 Vgl. Scheuerbrandt (w. o. Anm. 23) bes. 126.

30 Die Entfernung zwischen Butzbach und Ladenburg beträgt auf dem Fußweg entlang der heutigen Bundesstraße 3, die weitgehend mit der römischen Verbindungsstraße nach Süden korrespondiert, laut google-maps 117 km, was einem Fußmarsch von circa 24 Stunden entspricht (s. Abb. 9). 


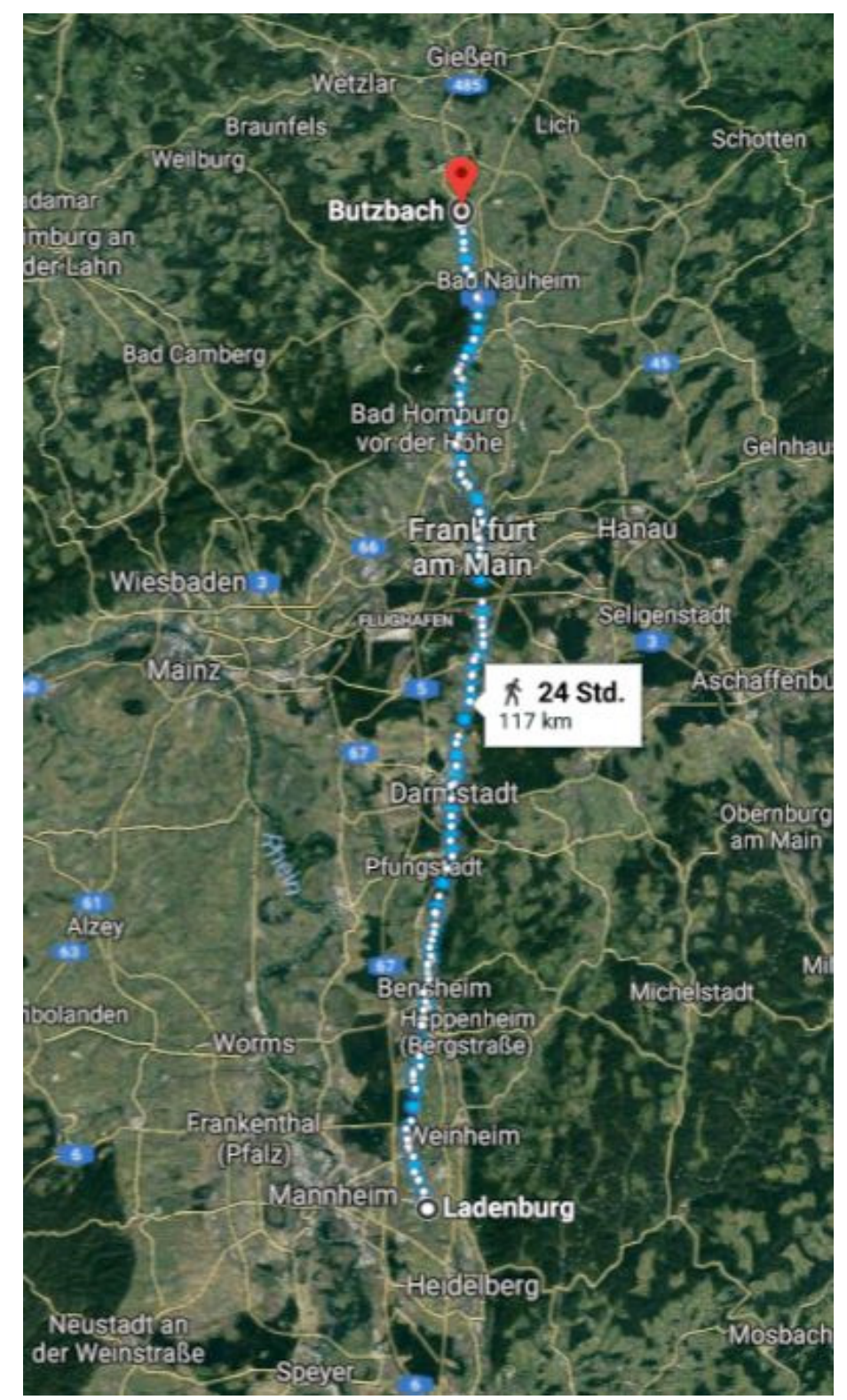

Abb. 9: Fußweg Butzbach - Ladenburg nach google-maps

Und doch verbleiben Ungereimtheiten im Befund der Inschrift, die zu benennen sind, auch wenn sie sich derzeit kaum ausräumen lassen werden: (Wie) ist es denkbar, dass ein junger Soldat, wie der hier bezeugte [---]us Magn[us], bereits in seinem ersten Dienstjahr als Soldat im Rang eines immunis die Funktion eines cornicen ausgeübt hat? Deren Aufgabe im Kampfgeschehen bestand im Verbund mit den signiferi in der zuverlässigen und eindeutigen Weitergabe der vom centurio ausgegebenen Befehle. Neben der Fertigkeit, dem cornu die geforderten Tonsignale zu entlocken, war hier maßgeblich auch eine hinreichende Kampfestauglichkeit gefragt. In der Tat sind nach Ausweis der - insgesamt vergleichsweise wenigen diesbezüglichen - epigraphischen Zeugnisse mit Lebens- und Dienstalterangaben cornicines im Alter zwischen 27 und 38 Jahren nach 4 bis 20 Dienstjahren gestorben. ${ }^{31}$ Selbstverständlich sagt dies nichts

31 Abfrage in EDCS und EDH nach ,,cornicen “ bzw. ,,cornicin “ + ,stip “ + Grabinschrift mit sieben Suchergebnissen (vom 04.05.2018): 1. ann. XXXVIII / stip. XVIII (PaI, CIL III 15159); 2. ann. XXXVI / stip. XVIII (GeI, CIL XIII 7779); 3. ann. XXXV/stip. XVII (Dal, ILJug 2811); 4. ann. 
über die jeweiligen Dienstalter bei Übernahme der Funktion als militärischer aenator aus. Das mit vier Jahren vergleichsweise geringe Dienstalter eines cornicen der stadtrömischen Prätorianersoldaten (s. u. Anm. 31 [6.]) deutet hier immerhin eine, sich vom nächst höheren Dienstalter von zehn Jahren deutlich absetzende, Tendenz an. Dennoch bleibt das mit sti. (scil. I) noch einmal niedrigere Dienstalter für einen cornicen bislang ohne Parallele. Ob nun die Möglichkeit einer solchen Funktion (und damit Lesung der Inschrift) bei einem militärischen Anfänger a priori auszuschließen ist, da sie als undenkbar gewertet wird, oder aber ob eine nach bestem Wissen und Gewissen als solche ausgemachte epigraphische Evidenz, trotz der in Kauf zu nehmenden Irregularitäten, bis zum Beweis des Gegenteils als Option zugelassen wird, ist eine Frage der Einschätzung und methodischen Herangehensweise. Vor dem Hintergrund der ohnehin nur sehr seltenen inschriftlichen Belege für cornicines (vgl. o. Anm. 1) neige ich dazu, vorerst einer , lectio difficilior' den Vorzug geben. ${ }^{32}$

\section{Kontakt zur Autorin:}

Dr. Francisca Feraudi-Gruénais

Epigraphische Datenbank Heidelberg (EDH)

Seminar für Alte Geschichte und Epigraphik

Marstallhof 4D - 69117 Heidelberg

Email: francisca.feraudi-gruenais@,zaw.uni-heidelberg.de

www.epigraphische-datenbank-heidelberg.de

\section{Abbildungsnachweise:}

Abb. 1: (C) Landesamt für Denkmalpflege am Regierungspräsidium Stuttgart, Dienstsitz Karlsruhe. Foto: Gunter Baumann

Abb. 2: CC BY-SA Francisca Feraudi-Gruénais, Kartenbasis

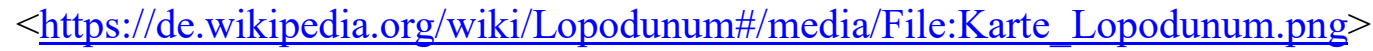

Abb. 3 und 4: CC BY-SA Francisca Feraudi-Gruénais (Skizze)

Abb. 5: CC BY-SA Francisca Feraudi-Gruénais (Umzeichnung)

Abb. 6: (wie Abb. 1); C Heidelberg - Kurpfälzisches Museum, Foto: Ortolf Harl 2016

Oktober $<$ http://lupa.at $/ 27110 /$ photos $/ 1>$

Abb. 7: CC BY-SA Francisca Feraudi-Gruénais (Collage), Kartenbasis

$<$ https://de.wikipedia.org/wiki/Lopodunum\#/media/File:Karte_Lopodunum.png>,

Fotos: (wie Abb. 1); (C) W. Strysio nach

$<$ https://edh-www.adw.uni-heidelberg.de/edh/foto/F004722>; (C) CIL XIII-Projekt

Flensburg/Trier

Abb. 8: nach Scheuerbrandt (w. o. Anm. 23), Taf. 11 (Ausschnitt)

Abb. 9: google-maps (Ausschnitt)

XXXII / stip. XI (Syr, AE 2005, 1558); 5. ann. triginta / stip. decem (Syr, AE 1993, 1594), allerdings ein tubicen, gestiftet von seinem Kollegen, einem cornicen; 6. ann. XXVII / stip. IIII (Roma, CIL VI 2724); 7. ann. [---] / stip. XX (PaS, AE 1990, 795).

32 Vielleicht weniger risikobehaftet, aber kaum befriedigender und letztlich mit nicht weniger offen bleibenden Fragen würde demgegenüber eine ,lectio facilior' in der Gestalt [D(is) M(anibus)](?) / [---] us Magn[us ---?] / [---]l(?)i (filius?) Bitur(igus) [---?] / [---? c]ornicen [---?] / [---?]TI(---) an(norum) XXV [---?] / [---? C] astia [---?] / ------?ausfallen; in Zeile 2 also unter Verzicht auf eine mögliche Kohortennennung und unter Ignorieren der mit großer Wahrscheinlichkeit auszumachenden Interpunktion vor I; ferner in Zeile 4 unter Verzicht auf die per Autopsie überprüfte sehr wahrscheinliche Ergänzung der oberen Serife zu einem S. 